\title{
The influence of cue type and configuration upon radial-maze performance in the rat
}

\author{
PHILIPP J. KRAEMER, MARY E. GILBERT, and NANCY K. INNIS \\ University of Western Ontario, London, Ontario, Canada
}

\begin{abstract}
The influence of cue type and cue configuration on radial-maze performance in rats was examined in two experiments. In the first experiment, it was found that rats provided with both salient intramaze and extramaze cues acquired the task faster than rats given only one set of cues. No difference in acquisition was found between a group trained with intramaze cues alone and a group trained with extramaze cues alone. In a cue-preference test, it was found that groups that had been trained with extramaze cues, intramaze cues, or both sets of cues relied on extramaze cues to avoid visited arms when given both types of cues concurrently. When all groups were transferred to intramaze-cue-alone trials, only the group that had been originally trained with extramaze cues alone showed any disruption. Also, during the second half of the intramazecue-alone trials, the arrangement of these cues was randomly changed on each trial. This disruption in cue configuration did not deleteriously affect performance in any of the three groups; all remained above chance performance, although the performance of the group originally trained with extramaze cues alone was inferior to that of the other two groups. In Experiment 2, groups of rats were trained on daily alternating trials under intramaze-cue-alone and extramaze-cuealone conditions. For one group, the configuration of intramaze cues was altered randomly on each trial; the other group had intramaze cues always presented in the same configuration over trials. It was found that acquisition was more rapid on intramaze trials in the group given static configurations. Also, acquisition of the extramaze task was faster than the intramaze task in the group given variable intramaze cue configurations. No difference was found between the intramaze and extramaze conditions in the group given static intramaze cue configurations. These data suggest that a static cue configuration may influence radial maze performance, but is not a necessary condition for such performance.
\end{abstract}

Since its introduction by Olton and Samuelson (1976), the radial maze has become a popular research tool for the study of memory processing in animals. The general phenomenon explored with this apparatus involves the organized and highly proficient foraging behavior demonstrated by rats searching for desired substances, such as food, placed on the maze. The maze itself consists of a circular central platform from which arms radiate like the spokes on a wheel. When rats are allowed to collect food located on the ends of the arms, a highly efficient pattern of collection soon emerges; they tend to choose unvisited arms before returning to previously visited arms. The available data seem to suggest that this behavior depends on memory processing rather than some other possible mechanism, such as response chaining, scent marking, or general reliance on odor cues (Maki, Brokofsky, \& Berg, 1979; Olton \& Samuelson, 1976; Zoladek \& Roberts, 1978).

Memorial accounts of radial-maze behavior have suggested that both working memory and reference

This research was supported by Natural Sciences and Engineering Research Council of Canada Grant A-9945 to N. K. Innis. The authors' mailing address is: Department of Psychology, University of Western Ontario, London, Ontario N6A SC2, Canada. memory are involved. Working memory refers to the retention of information necessary for accurate performance within a specific trial on some task. Reference memory corresponds to the retention of information necessary for accurate performance on all trials of a task (Honig, 1978). For the radial maze, a trial typically consists of allowing the animals to obtain all available rewards from the ends of the arms, or to make a fixed number of arm choices. Working memory for radial-maze performance would involve memorial representations enabling the subject to avoid reentering previously visited arms during a trial. The reference memory component would involve the representation of information, such as the spatial layout of the maze and general characteristics of the problem, which could be used on all trials.

The idea that the spatial layout of the maze may be represented in memory and may be influential in directing arm choices represents a revival of Tolman's (1948) concept of cognitive mapping. It has been argued that the arms of the maze become identified with spatial locations, and a cognitive map of these locations is stored in reference memory. Representations of visited arms are held in working memory during each trial, and are used in conjunc- 
tion with the cognitive map to avoid revisiting these locations (O'Keefe \& Nadel, 1978; Olton \& Samuelson, 1976; Roberts, 1979; Roitblat, 1982).

Research has shown that spatial locations are normally identified by extramaze cues rather than intramaze cues (Dale, 1979; Olton \& Collison, 1979; Olton \& Samuelson, 1976; Walker \& Olton, 1979). In addition, it has been found that rats seem to respond to the overall configuration of extramaze cues, and do not approach or avoid discrete arm cues (Suzuki, Augerinos, \& Black, 1980). Under certain conditions, however, rats can learn to rely on nonspatial intramaze information to avoid visited arms. Olton, Becker, and Handelman (1979) described a study in which rats were trained on a four-arm covered maze. Visual-tactile inserts were placed along the floors of the arms, and the spatial configuration of these cues was altered between choices and over trials. It was found that the rats were able to avoid revisits to specific arms during a trial, although they took longer to reach criterion than a group given statically arranged extramaze cues.

The current study attempted to investigate further the influence of intramaze and extramaze cues, as well as the configurational arrangements of these cues, on radial-maze performance in rats. The general question of interest concerned how radial-maze performance might be affected by the nature of the cues that identify the arms of the maze.

\section{EXPERIMENT 1}

Phase 1 of Experiment 1 examined the relative rates of acquisition for various cue conditions. One group was provided with explicit extramaze cues consisting of the environmental stimuli surrounding the maze. A second group was given explicit intramaze cues consisting of visual-tactile inserts placed along the floors of each arm. For this group, the maze was covered throughout training so that salient extramaze visual information was drastically reduced. A third group had both sets of cues present during acquisition. These subjects were trained with the maze uncovered and the intramaze inserts in place. In Phase 2 of the experiment, all subjects were tested with both sets of cues available to determine any cue preferences that may have developed during acquisition. On each trial, the maze was rotated after the subject's third arm choice, so that the correlation between extramaze locations and intramaze cues was disrupted. Performance was assessed in terms of both the number of visits, following rotation, to different arms and extramaze locations. Any cue preference would have appeared as a tendency to visit either different arms or different extramaze locations. These tendencies were compared between groups with respect to their acquisition experience. Phases 3 and 4 consisted of testing all groups with the maze covered and intramaze cues available. During Phase 3, the intramaze cues were arranged in the same configuration that had been used in Phases 1 and 2. For Phase 4 , these cues were rearranged randomly on each trial. Performance of the three groups under these conditions was again examined with respect to their original acquisition experience.

\section{Method}

Subjects. Twenty-one adult male hooded rats of the Long-Evans strain served as subjects. The animals were housed individually in standard wire cages and permitted free access to food and limited access to water. Each subject was placed on a $231 / 2-h$ waterdeprivation schedule, reduced to $85 \%$ of its ad-lib weight through water rationing, and maintained at that level throughout testing. Water rations were provided at the end of each daily session.

Apparatus. During the pretraining phase of the experiment, a straight alley was employed. This consisted of a wooden runway, $8.5 \mathrm{~cm}$ wide and $122 \mathrm{~cm}$ long. The runway was surrounded by $16-\mathrm{cm}$-high walls and was painted flat gray. The entire structure was located in a room adjacent to that used for the radial maze. A single water receptacle was placed at one end of the alley. This consisted of a shallow 3-cm-diam plastic cup enclosed in a metal food receptacle measuring $7.5 \mathrm{~cm}$ long $\times 5 \mathrm{~cm}$ wide $\times$ $2.5 \mathrm{~cm}$ deep.

Experimental testing was conducted on a partially enclosed radial maze. This wooden structure was painted flat gray and consisted of a central platform, $31.5 \mathrm{~cm}$ in diameter, with eight arms radiating outward $45 \mathrm{deg}$ from one another. Each arm was $80 \mathrm{~cm}$ long, $10 \mathrm{~cm}$ wide, and surrounded by $16.5-\mathrm{cm}$-high walls. A water receptacle, identical to the one described above, was placed at the end of each arm. The maze was placed on a circular rotating stool which permitted easy rotation in clockwise or counterclockwise direction. Photocell recording devices were located $16 \mathrm{~cm}$ from the end of each arm of the maze. These consisted of a $1.5-\mathrm{cm}$ hole bored into one side wall, with a 32-W light bulb mounted on the outside of this hole. This projected a light beam onto the opposite inside wall of the arm. On this wall was hung a $12.8 \times$ $3.5 \times 2.1 \mathrm{~cm}$ block of wood containing a $.8-\mathrm{cm}$ photocell recessed in the $2.1-\mathrm{cm}$ face of the block. Each photocell was wired to a separate channel of an event recorder. The pens of this recorder were operated by interruptions in the light beam to each photocell. These pen deflections served as the index of the subjects' arm choices during a trial. The event recorder was located in a room adjacent to the testing room.

The experimental room contained a number of distinct stimulus objects which surrounded the maze and provided the salient extramaze visual cues. These included: a fluorescent light fixture and air ventilation duct in the ceiling, a sink, window, and door. Intramaze cues were provided by visual-tactile inserts that could be placed along the arm floors. These consisted of a variety of materials which differed visually and tactually and included: sandpaper, plywood, fiber board, sheet metal, wire mesh, rubber foam, shag carpet, and rubber carpet underpadding. These inserts covered the entire length of an arm and extended $3 \mathrm{~cm}$ onto the center platform. For trials in which the maze was covered, eight translucent plastic panels were placed on top of the walls of each arm. These allowed diffuse light from the overhead light fixture to illuminate the maze without providing access to discrete extramaze visual cues. The center platform was covered with a circular cardboard disk that was put in place after a subject had been released onto the maze.

Procedure. The pretraining phase of the experiment consisted of five daily trials in which the subjects were individually released onto the straight alley and allowed to consume $.2 \mathrm{ml}$ of tap water from the water receptacle located at one end of the alley. These trials continued until the subjects ran readily to the end of the alley 
containing the water. Experimental testing began on the following day.

Following pretraining, the subjects were divided randomly into three groups of seven animals each. Each animal was tested on one trial per day. On each trial, the subject was released onto the central platform and allowed to make eight arm choices, as defined by the photocell recording device. Throughout the experiment, the animals were released onto the central platform facing one of the eight arms according to a random order. Prior to the start of each trial, the eight water receptacles were baited. Both arm choices and total trial durations were recorded.

Each group received a different set of cue conditions during acquisition. Group I received training with intramaze cues available. For these subjects, the intramaze cue inserts were in place and the maze was covered. Group $\mathrm{E}$ was given training with only extramaze cues; the visual-tactile inserts were removed and the maze was left uncovered. Group IE received both intramaze and extramaze cues; the maze was uncovered and the visual-tactile inserts were in place. Criterion was set at three consecutive trials in which no more than a total of one arm repetition occurred within the three trials. Following Phase 1 , subjects in all three experimental conditions were treated identically.

During Phase 2, each subject was given daily trials with both intra- and extramaze cues present (i.e., the intramaze cue inserts were in place and the maze was uncovered). The trials of Phase 2 alternated daily between control and test trials for a total of 10 trials of each type. During both types of trials, the subjects were removed from the maze after their third arm choice and placed in their home cage. On control trials, the maze was rotated 360 deg during the subjects' absence, such that the alignment of intramaze cues and extramaze locations was the same before and after rotation. The subjects were released again onto the central platform following maze rotation and allowed to make an additional five choices. For test trials, the same general procedure was followed, except that the maze was rotated such that the alignment of intramaze cues and extramaze locations was different after rotation from what it had been during the initial three arm choices. The rotation was always of the same type; that is, the three visited intramaze cues were realigned with three unvisited extramaze locations, while three of the unvisited intramaze cues were aligned with three visited extramaze locations. Such an arrangement was always possible, regardless of the distribution of the three initial choices. Given that no arms are repeated in the first three arm choices and the geometrical constraints of an eight-arm radial maze, the relative spatial distributions of three arm choices are limited to the patterns illustrated in Figure 1. For each possible pattern, maze-rotation alternatives that would satisfy the condition that no previously visited intramaze cue be aligned with a previously visited extramaze location are limited to those values presented in the figure. The actual degree of maze rotation for each test trial was determined by the experimenter after a subject's third arm choice. On each test trial, the pattern of the first three choices was compared with the possible patterns presented in Figure 1, and a rotation alternative was chosen from a random serial list for that pattern. As a rotation alternative was chosen, it was eliminated from the list so that the next occurrence of that pattern resulted in a different rotation alternative. Figure 2 provides an example of such a rotation. The top panel of the figure illustrates the intramaze cues (numbered 1-8) and extramaze locations (lettered A-H) associated with each arm. The solid triangles indicate the intramaze cues and extramaze locations visited during the first three choices of a hypothetical trial. For the distribution of choices illustrated in the example, only a maze rotation of $180 \mathrm{deg}$ would result in each of the three visited intramaze cues being aligned with three unvisited extramaze locations, and likewise for the three initially visited extramaze locations. The alignment of intramaze cues and extramaze locations after a 180-deg rotation is illustrated in the lower panel of the figure. The total time the animal was absent from the maze during rotation was always less than $60 \mathrm{sec}$ for both control and test trials.

\section{Choice Distributions \\ Rotation Alternatives}
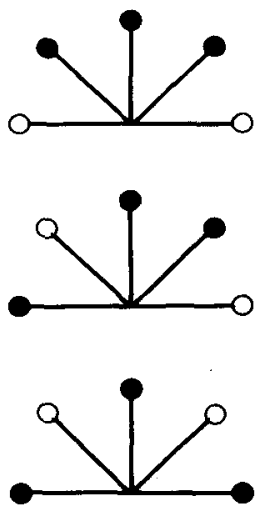

$45^{\circ} 135^{\circ} 225^{\circ} 315^{\circ}$

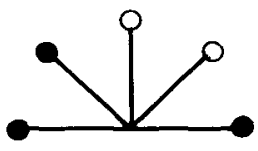

\section{$135^{\circ} 180^{\circ} 225^{\circ}$}

$180^{\circ}$
Figure 1. Possible distributions of the three initial arm choices and rotation alternatives used in Phase 2.

Arm choices were recorded in terms of both visits to extramaze locations and intramaze cues.

For Phase 3, all subjects were tested on five trials with the visualtactile inserts in place and the maze covered. The configuration of the intramaze cues was the same as when these cues had been present in the previous phases. Phase 4 consisted of five additional trials under the same cue conditions except that the configuration of the visual-tactile inserts was varied randomly on each of the five trials. The only restriction on these configurations was that the original arrangement of the previous phases was not used.

\section{Results and Discussion}

Analysis of trial durations revealed no significant differences between conditions in any phase of the experiment. Therefore, these data will not be discussed further.

Phase 1. Rates of acquisition for the three groups were compared in terms of trials to criterion. The mean trials to criterion for the IE, I, and E groups were $9.1,15.0$, and 17.1 , respectively. These means were found to differ significantly $[F(2,18)=8.97$, $\mathrm{p}<.004]$. Individual group comparisons revealed that the IE group reached criterion in significantly fewer trials than either the I group $[t(12)=3.02, p<$ $.02]$ or the E group $[t(12)=4.10, \mathrm{p}<.01]$, which did not differ from each other $[t(12)=1.07, p>.3]$.

In order to assess any possible differences in response strategies used by the three groups during acquisition, turn magnitudes for each group were analyzed. A choice of an arm adjacent to the arm being departed counted as a turn magnitude of 1 . Similarly, an arm choice $90 \mathrm{deg}$ from the departed arm counted as an arm magnitude of 2 . Accordingly, 


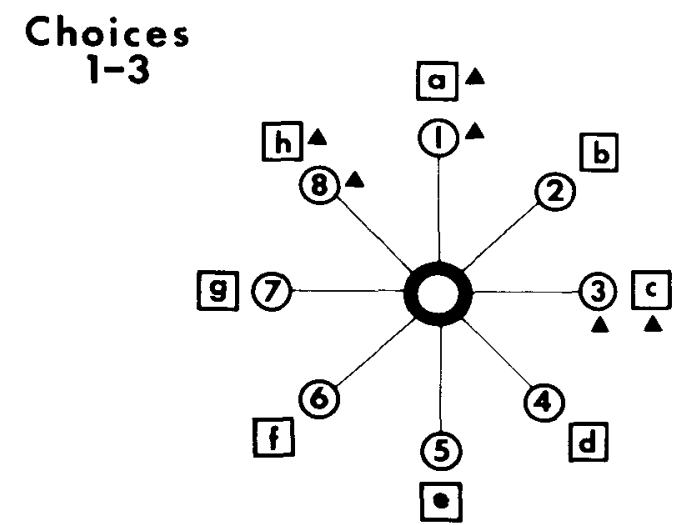

\section{Choices 4-8}

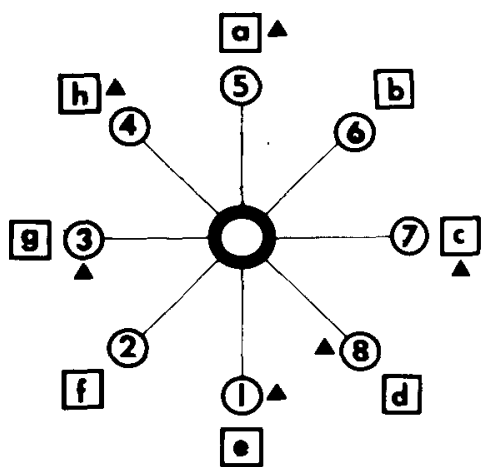

Figure 2. Example of intramaze and extramaze cue alignment before and after a 180-deg rotation.

turn magnitudes of 1-4 are possible (Yoerg \& Kamil, 1982). The mean turn magnitudes for each group on the last three trials of acquisition were compared, and no significant differences were obtained. Thus, the acquisition-rate differences between groups could not be accounted for in terms of differences in response patterns.

These data suggest that the availability of two redundant sets of information, which could be used to identify individual arms or arm locations, facilitated radial-maze acquisition; the IE group learned faster than either of the single-cue groups. This finding is consistent with other research on animal learning in which it has been consistently found that the presence of multiple cues enhances discrimination learning (Restle, 1957; Sutherland \& Macintosh, 1971). A somewhat unexpected finding was that the rates of acquisition for the I and $E$ groups were comparable. Most of the available data have shown that rats typically rely on extramaze information in solving the radial maze task (Olton \& Collison, 1979; Olton \& Samuelson, 1976; Roitblat, 1982; Walker \& Olton, 1979). Thus, one might infer that there is something about extramaze cues that renders them particularly well suited for identifying and avoiding visited arms.
If this were the case, it would be reasonable to expect that reliance on such cues might lead to more rapid acquisition, relative to reliance on the less preferred intramaze cues. In the present situation, however, radial maze proficiency was acquired equally well for rats given either salient intramaze cues or salient extramaze cues.

Phase 2. Performance during the cue-preference phase of testing is represented in Figure 3, which shows the mean different intramaze cues and extramaze locations visited during control and test trials. It should be noted that no arm repetitions were recorded for any of the subjects during the three initial arm choices. The data for the control trials represent performance when the alignment of intramaze cues and extramaze locations was the same before and after rotation. For all three groups, the mean number of different arms chosen remained well above chance throughout these trials (chance $=5.3$; Bond, Cook, \& Lamb, 1981; Olton, 1978). The overall mean number of different arms visited on the control trials for the IE, I, and E groups were 7.6, 7.3, and 7.3 , respectively. Thus, performance on control trials did not differ across groups or over trials (see Figure 3). Also, performance was not disrupted by mere removal from the maze during rotation, or by the change in conditions experienced by Groups I and $\mathrm{E}$.

Data from the test trials, during which the correlation between intramaze and extramaze cues was disrupted following maze rotation, are also presented in Figure 3. The eight arm choices (including the first three choices prior to maze rotation) are plotted separately in terms of the mean number of visits to different extramaze locations and different intramaze cues. Analyses revealed that each group chose a greater number of different extramaze locations than different intramaze cues over the 10 test trials [Group I, $F(1,6)=7.96, \mathrm{p}<.05$; Group IE, $F(1,6)=$ 98.9, p <.001; Group E, $F(1,6)=24.3$, p < .05]. Both Group IE and Group E showed a clear preference for avoiding revisits to extramaze locations, but returned to previously visited intramaze cues throughout the 10 test trials. This preference, however, was less pronounced in Group E. Also, as the figure indicates, a clear preference for choosing different extramaze locations over different intramaze cues in Group I did not emerge until the later trials of testing, although a significant interaction between cue choice and trials was not obtained.

The data from Phase 2 indicate that the subjects were primarily avoiding revisits to extramaze locations, rather than specific intramaze cues. This tendency was apparent from the onset of testing for the IE and $E$ groups. It is interesting that animals continued to respond in this way despite the inefficiency of the strategy. Water rewards were rotated with the intramaze cues, and these cues were more reliable predictors of reward than the extramaze locations. 


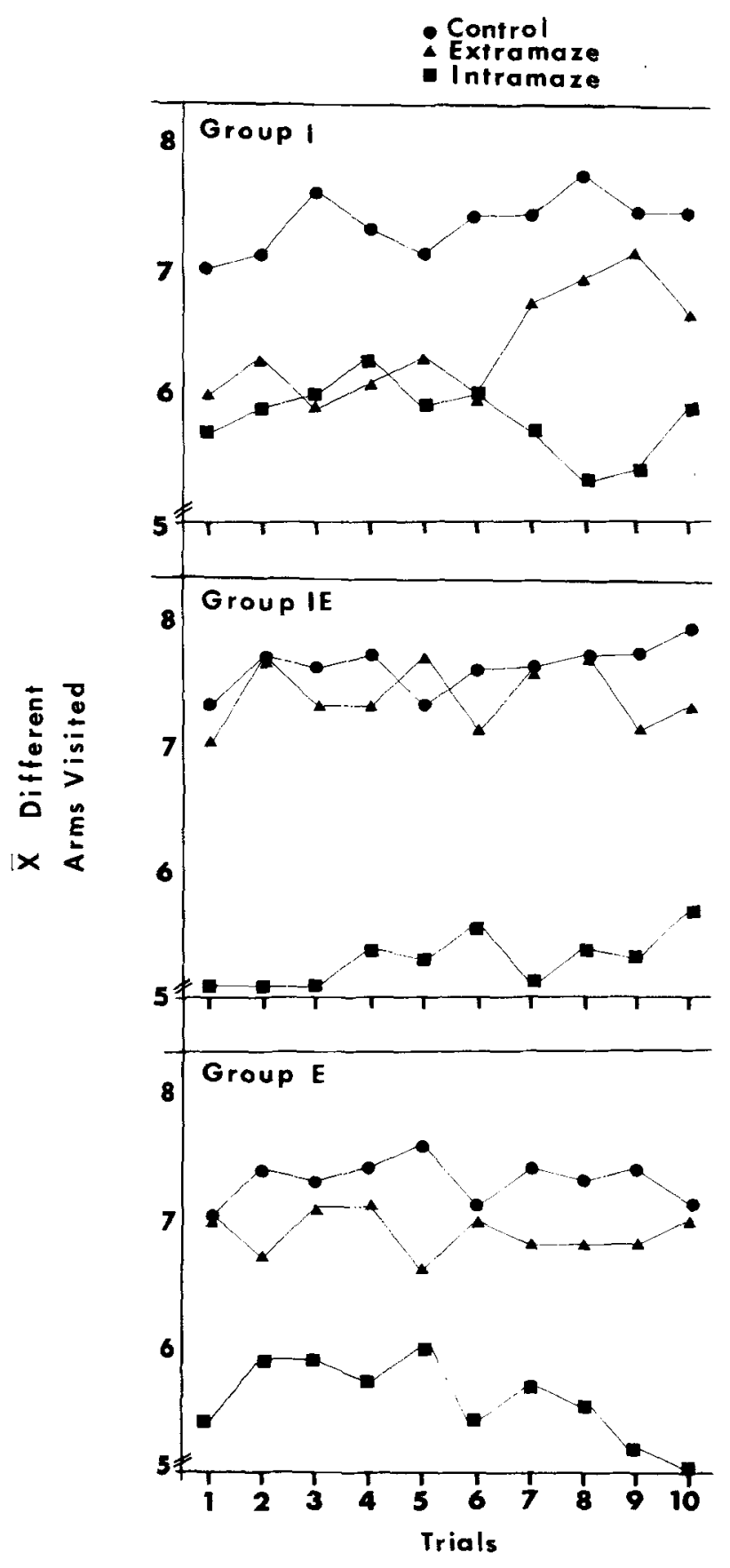

Figure 3. Mean different arms visited on control and preference test trials during Phase 2.

The performance of the I group shows that even these subjects, initially trained with intramaze cues, were not responding in terms of these cues when provided with both intramaze cues and extramaze cues. The lack of a clear preference for either set of cues on the early trials of testing for this group may have been the result of competition between extramaze and intramaze cues for the control over arm choices. The eventual preference for avoiding extramaze locations, which emerged on the final trials of testing, could have resulted from performance finally having come under the control of extramaze cues.

Phases 3 and 4. The results from Phase 3 are presented in the lower panel of Figure 4 . The mean number of different arms visited is plotted over the five trials for all three groups. It can be seen that performance for the I and IE groups remained well above chance on all five trials in which only intramaze cues were present, but arranged in the familiar configuration experienced in the previous phases. The $E$ group, however, showed a deficit on the early test trials. Their performance then increased to a level well above chance on Trials 4 and 5 . A mixed analysis of variance was performed on these data, which included the factors groups and trials. A significant main effect was obtained for groups $[F(2,18)=19.0$, $\mathrm{p}<.001]$ and trials $[F(4,72)=3.40, \mathrm{p}<.02]$. No significant trials $\times$ groups interaction was obtained. Individual group comparisons revealed that both the $I$ and IE groups were significantly superior to the $E$ group $[\mathrm{t}(12)=2.34, \mathrm{p}<.001$, and $\mathrm{t}(12)=2.52, \mathrm{p}<$ .001 , respectively]. The I and IE groups did not differ significantly $[\mathrm{t}(12)=.234, \mathrm{p}>.5]$.

These results indicate that both the I and IE groups performed quite accurately when transferred to the intramaze cue-only condition. The performance of the I group is not at all surprising, since these subjects originally had acquired the task with only intramaze cues available. The more interesting finding concerns the IE group. These subjects had never before experienced the intramaze cues in isolation and had shown no tendency to avoid visited intramaze cues when tested in Phase 2. Nonetheless, they were able to avoid reentering visited arms as accurately as the I group when given only intramaze cues. This suggests that their prior experience with the intramaze cues, including both acquisition and phase 2 , was sufficient to allow them to rely effectively on these cues in isolation. That it was primarily the acquisition experience that allowed the IE group to transfer effectively to the intramaze cue-only condition can be inferred from the performance of the $E$ group. Since these subjects had had the same experience with intramaze cues during Phase 2 as the IE group, it would appear that it was the differential acquisition experience with these cues that was responsible for the difference between the two groups in Phase 3.

The data from Phase 4 are presented in the upper panel of Figure 4. The mean numbers of different arms visited were subjected to a mixed analysis of variance, with groups and trials as factors. Significant differences were found between groups $[F(2,18)$ $=10.83, \mathrm{p}<.001]$. The effects of trials and the groups 


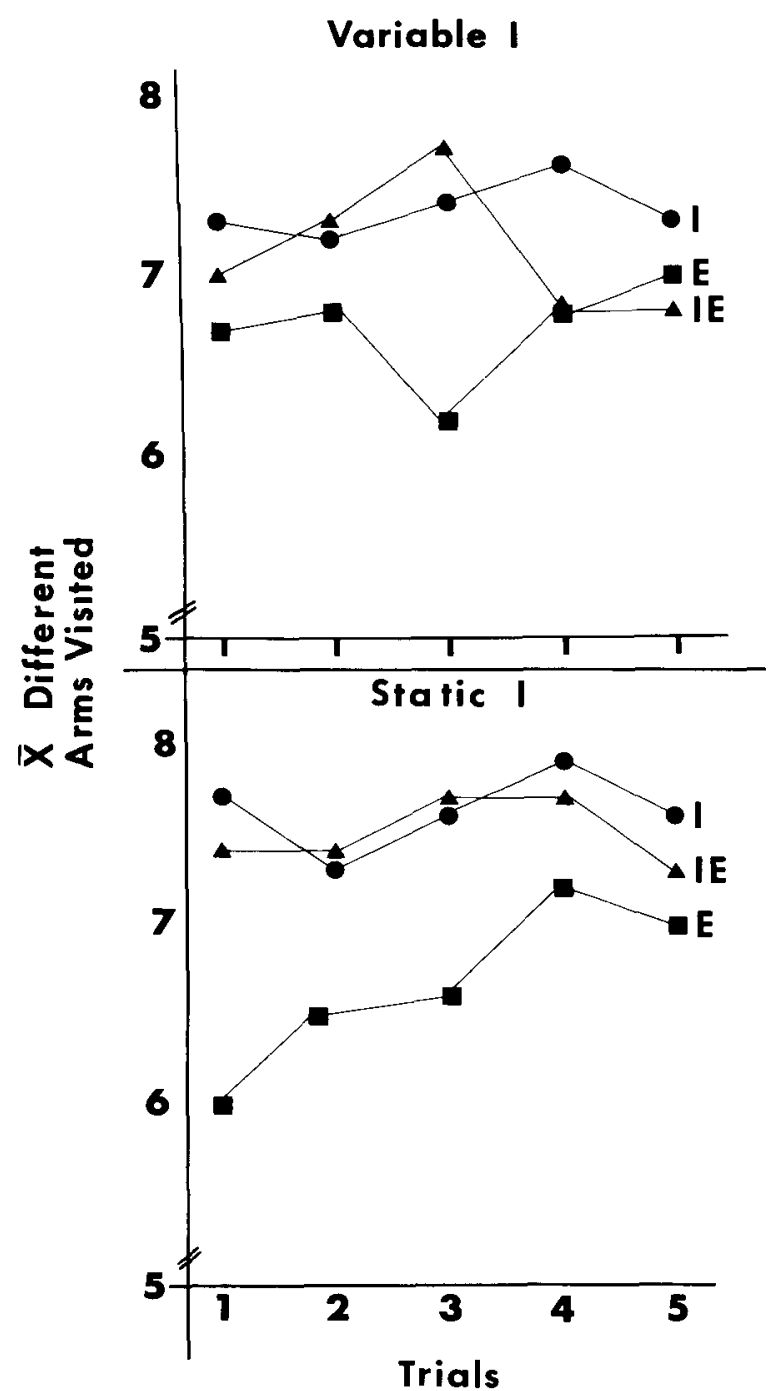

Figure 4. Mean different arms visited during Phases 3 and 4.

$\times$ trials interaction did not reach significance. Individual group comparisons revealed that the I and IE groups were significantly superior to the $E$ group $[\mathrm{t}(12)=1.25, \mathrm{p}<.02$, and $\mathrm{t}(12)=2.05, \mathrm{p}<.001$, respectively], while the I and IE groups did not differ from each other $[t(12)=.79, p>.05]$.

Figure 4 shows that the overall performance in all three groups remained well above chance throughout Phase 4, although performance was somewhat better in Groups I and IE than in Group E. These data are consistent with results reported by Olton et al. (1979). It is clear that rats are able to avoid revisiting discrete maze arms, even when the intramaze cues associated with the arms are rearranged randomly across trials.

\section{EXPERIMENT 2}

In Experiment 1, it was found that rats could learn to perform effectively on the radial maze task in the presence of either intramaze or extramaze cues. Experiment 2 attempted to examine further how acquisition might be influenced by these two types of cues. Two groups of rats were given trials that alternated daily between intramaze-only and extramaze-only cue conditions. For one group (Group IS), the intramaze cues were arranged statically on all of the intramaze-cue trials. For the other group (Group IR), the intramaze cues were rearranged randomly on each trial. Both groups of subjects were treated identically during the extramaze-cue trials; that is, the intramaze-cue inserts were removed and the extramaze cues remained in the same configuration for both groups. This design permitted examination of the relative rates of acquisition under intramaze- and extramaze-cue conditions experienced by the same subject. Also, Experiment 1 had revealed positive transfer to a condition in which intramaze cues were rearranged randomly over trials. All groups, however, had originally acquired the task with a static configuration of cues. Whether cue configurations might influence acquisition was examined in this experiment. The difference between intramaze-staticand intramaze-random-cue conditions was assessed both directly (i.e., the rates of acquisition during intramaze-cue conditions for Groups IS and IR) and relative to performance by the same subjects on trials with extramaze cues.

\section{Method}

Subjects. Twenty adult male hooded rats of the Long-Evans strain served as subjects. All animals were housed individually in standard wire animal cages contained in a common animal housing unit. The subjects were maintained on a free-food diet with limited access to water. Prior to pretraining, the subjects were reduced to $85 \%$ of their ad-lib weights through water rationing, and maintained at that level throughout the experiment. Daily water rations were provided at the end of each session.

Apparatus and Procedure. The maze, intramaze-cue inserts, and experimental testing room were the same as those used in Experiment 1 . Following the same pretraining experience used in Experiment 1 , the animals were assigned randomly to one of two groups of 10 subjects. Each group was given acquisition training consisting of alternating daily trials with intramaze and extramaze cues. On a given day, the subject was trained either with the intramaze-cue inserts in place and the maze covered (I trials) or with the intramaze-cue inserts removed and the maze left uncovered ( $E$ trials). For Group IS, the arrangement of the intramaze cues remained static over trials, while the arrangement was varied randomly across trials for the IR group. Trial type was alternated regularly over days. Half the subjects in each group began acquisition with $E$ trials, and the other half with I trials. This sequence of testing was continued for both groups until criterion was achieved on both trial types. This meant that if criterion was reached on one trial type before it was reached on the other, the daily alternation of trial type was continued until criterion was met for both. Criterion was again three consecutive trials in which no more than a total of one arm choice was repeated.

\section{Results and Discussion}

The mean trials to criterion for Group IS on I and E trials were 7.8 and 10.0, respectively; for Group IR they were 11.0 and 7.6 , respectively. A mixed analysis of variance revealed a significant interaction be- 
tween groups and trial type $[F(1,18)=8.4, p<.01]$. Individual comparisons indicated that Group IS took significantly fewer trials to reach criterion on I trials than Group IR $[t(18)=2.3, p<.05]$. In addition, Group IR reached criterion significantly sooner during $E$ trials than during I trials $[\mathrm{t}(9)=2.5, \mathrm{p}<.05$ ] No significant differences were obtained between Groups IS and IR for E trials, or between I and E trials for Group IS.

These data indicate that acquisition was influenced by the configuration of intramaze cues. Acquisition was more rapid in the presence of statically arranged intramaze cues than when these cues were presented in different configurations over trials. Nonetheless, it is clear that while static configurations may influence acquisition, they are not a necessary condition for learning to occur. Group IR did reach criterion even when the intramaze-cue configurations did not remain constant. Also, when the intramaze cues were statically arranged, acquisition for the same subjects was comparable under intramaze- and extramaze-cue conditions. This finding is consistent with data from Experiment 1, in which the same effect was observed between groups trained with only one set of cues.

\section{GENERAL DISCUSSION}

It has generally been assumed that rats rely on extramaze cues to avoid previously visited arms on the radial maze (Olton \& Samuelson, 1976; Roitblat, 1982). The results of the present experiments suggest, however, that conspicuous extramaze cues are not a necessary condition for accurate performance. When salient intramaze cues are provided, rats can effectively rely on these cues to discriminate visited and unvisited arms in the absence of salient extramaze visual cues. A similar finding has been reported by Olton et al. (1979). Moreover, when intramaze cues remain in a static configuration over trials, the rates of acquisition with either intramaze or extramaze cues are equivalent. Nonetheless, the current data also revealed that extramaze cues may be preferred to intramaze cues. In Experiment 1, groups trained with either extramaze cues alone or both extramaze cues and salient intramaze cues tended to avoid previously visited extramaze locations rather than visited intramaze cues. In addition, even a group originally trained with only intramaze cues failed to continue responding to these cues when confronted with both intramaze and extramaze cues; they eventually developed a preference for extramaze cues, even though these cues were less reliable predictors of reward and were less familiar. One possible explanation for this prevalent preference for extramaze cues might involve the discriminability of the two types of cues. Perhaps extramaze cues are easier for the rats to distinguish when discriminating previously visited arms on the maze. If this were the case, however, one might expect acquisition under an intramaze-cueonly condition to be retarded relative to an extramazecue-only condition. No such effect was obtained in the present experiments. Therefore, it is possible that some other mechanism may be responsible for the observed extramaze-cue preference. This hypothesis might best be evaluated through parametric variation in the discriminability of intra- and extramaze cues.

Another theoretical position adopted by many researchers is the notion that rats form cognitive maps, which they then use to avoid revisiting arms on the maze (O'Keefe \& Nadel, 1978; Olton, 1978; Roitblat, 1982). An implication of this hypothesis is that performance will be influenced by the cue configurations used by rats choosing arms to be entered or avoided. It has been shown that when extramazecue configurations are altered, performance deteriorates (Suzuki et al., 1980). The data presented here, as well as those reported by Olton et al. (1979), suggest, however, that static-cue configurations are not a necessary condition for accurate performance on the radial maze. Subjects trained with intramaze cues that were randomly rearranged over trials did achieve a high level of accuracy in avoiding previously visited arms, although they took slightly longer to reach criterion than a group trained with statically arranged intramaze cues (Experiment 2). Also, subjects trained with a static-intramaze-cue configuration were able to transfer effectively to a condition in which the intramaze cues were presented in changing configurations over trials (Experiment 1). These findings may question the cognitive map hypothesis. If it is assumed that rats form cognitive maps that represent configurations of intramaze or extramaze cues associated with arms on a radial maze, then a static configuration across trials would seem to be a necessary condition for the formation of such representations. Evidence of accurate performance in the absence of a static configuration challenges this hypothesis. It is possible that rats may not simply represent specific intra- or extramaze cue configurations, but may form general directional maps. These representations would provide directional, rather than locational, information and could then be used by the rats in conjunction with specific extra- or intramaze cues to orient when placed on the maze. Therefore, rats may be avoiding arms associated with relative directions rather than locations or discrete cues.

Another possibility is that rats may employ other types of representations. For example, they may represent the arms of the maze as discrete cues in some listlike fashion. With such a representation, they could tag visited arms during a given trial and use such tags to discriminate visited from unvisited arms. It remains to be shown under what conditions such nonmaplike representations could be used, as well as the storage capacity and retrieval efficiency for such representations. 
Finally, it has been suggested that the proficient choice performance observed in radial maze experiments may be related to the natural foraging characteristics of the rat (Bond, Cook, \& Lamb, 1981; Yoerg \& Kamil, 1982). It has been argued that the tendency for rats to avoid previously visited arms may be related to a general win-shift strategy observed in their food foraging behavior. Such a general strategy may differ for different types and degrees of deprivation, as well as for the type of reward obtained on the maze (Bolles \& Petrinovitch, 1956; Olton \& Schlosberg, 1978). Most of the available research has involved the use of food-deprived rats searching for food placed on the arms of the maze. It is interesting, therefore, that highly proficient choice accuracy was demonstrated for water-deprived animals searching for water rewards in the current experiments. These data are consistent with the findings obtained by Dale and Roberts (Note 1), in which radial maze performance was assessed factorially in terms of type of deprivation (food vs. water) and degree of deprivation ( $85 \%$ vs. $100 \%$ of body weight). Dale and Roberts found that water-deprived subjects searching for water rewards effectively learned to avoid returning to previously visited arms and, in fact, acquired such proficiency faster than food-deprived animals searching for food reward, irrespective of the degree of deprivation. Thus, the suspicion that accurate choice performance on the radial maze is dependent upon general behavioral characteristics associated with food foraging may not be entirely accurate. Obviously, continued research into this question is required.

\section{REFERENCE NOTE}

1. Dale, R. H. I., \& Roberts, W. A. Variations in radial-maze performance under different deprivation and reward conditions. Unpublished manuscript, University of Western Ontario, 1983.

\section{REFERENCES}

Bolles, R. C., \& Petrinovitch, L. Body-weight changes and behavioral attributes. Journal of Comparative and Physiological Psychology, 1956, 49, 177-180.

Bond, A. B., Cook, R. G., \& Lamb, M. R. Spatial memory and the performance of rats and pigeons in the radial-arm maze. Animal Learning \& Behavior, 1981, 9, 575-580.

DALE, R. H. I. The role of vision in the rat's radial maze performance. Unpublished doctoral dissertation, University of Western Ontario, 1979.

Honia, W. K. Studies of working memory in the pigeon. In S. H. Hulse, H. Fowler, \& W. K. Honig (Eds.), Cognitive processes in animal behavior. Hillsdale, N.J: Erlbaum, 1978.

Mackintosh, N. J. The psychology of animal learning. New York: Academic Press, 1974.

Maki, W. S., Brokofsky, S., \& Berg, B. Spatial memory in rats: Resistance to retroactive interference. Animal Learning \& Behavior, 1979, 7, 25-30.

O'KeEFE, J., \& NADEL, L. The hippocampus as a cognitive map. Oxford: Clarendon Press, 1978.

Otton, D. S. Characteristics of spatial memory. In S. H. Hulse, H. Fowler, \& W. K. Honig (Eds.), Cognitive processes in animal behavior. Hillsdale, N.J: Erlbaum, 1978.

Olton, D. S., Becker, J. T., \& Handelman, G. E. Hippocampus, space and memory. Behavioral and Brain Sciences, $1979,2,313-365$.

Olton, D. S., \& Collison, C. Intramaze cues and "odor trails" fail to direct choice behavior on an elevated maze. Animal Learning \& Behavior, 1979, 7, 221-223.

Olton, D. S., \& SAmuelson, R. J. Remembrances of places passed: Spatial memory in rats. Journal of Experimental Psychology: Animal Behavioral Processes, 1976, 2, 97-116.

Olton, D. S., \& Schloseng, P. Food-searching strategies in young rats: Win-shift predominates over win-stay. Journal of Comparative and Physiological Psychology, 1978, 92, 609-618.

RESTLE, F. Discrimination of cues in mazes: A resolution of the 'place-vs-response' question. Psychological Review, 1957, 64, 217-228.

RoBerts, W. A. Spatial memory in the rat on a hierarchical maze. Learning and Motivation, 1979, 10, 117-140.

Roitblat, H. L. The meaning of representation in animal memory. Behavioral and Brain Sciences, 1982, 5, 353-406.

Sutherland, N. S., \& Mackintosh, N. J. Mechanisms of animal discrimination learning. New York: Academic Press, 1971.

Suzuki, S., Augerinos, G., \& Black, A. H. Stimulus control of spatial behavior on the eight-arm maze in rats. Learning and Motivation, 1980, 11, 1-8.

Tolman, E. C. Cognitive maps in rats and men. Psychological Review, 1948, 55, 189-208.

Walker, J. A., \& Olton, D. S. The role of response and reward in spatial memory. Learning and Motivation, 1979, 10, 73-84.

Yoeng, S. J., \& Kamil, A. C. Response strategies in the radial arm maze: Running around in circles. Animal Learning \& Behavior, 1982, 10, 530-534.

ZoladeK, L., \& Roberts, W. A. The sensory basis of spatial memory in the rat. Animal Learning \& Behavior, 1978, 6, 77-81.

(Manuscript received January 4, 1983 ;

revision accepted for publication June 2,1983 .) 